\title{
Comparison of change detection methods based on the spatial chaotic model for synthetic aperture radar imagery
}

\author{
Chih-Hsuan Huang ${ }^{1, *}$, Hsuan Ren ${ }^{1}$, and Yu-Chang Tzeng ${ }^{2}$ \\ ${ }^{1}$ Center for Space and Remote Sensing Research, National Central University, Taoyuan City, Taiwan \\ ${ }^{2}$ Department of Electronic Engineering, National United University, Miaoli, Taiwan
}

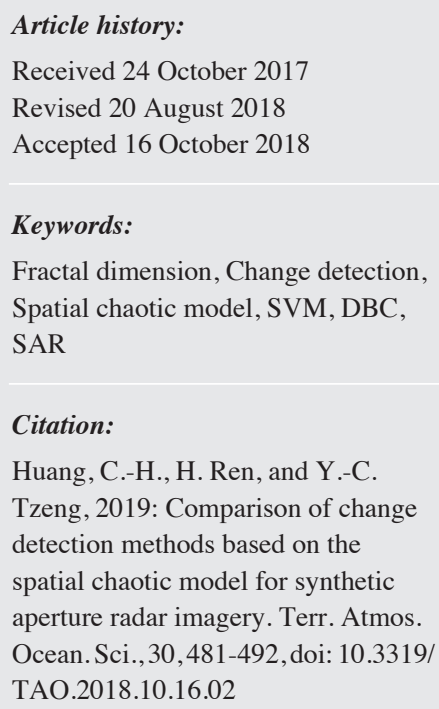

\begin{abstract}
Due to their all-weather, all-time and penetration characteristics, synthetic aperture radar (SAR) images are frequently used to monitor ground targets. As a result, environmental changes via natural events or human activities can be observed by applying a change detection technique. Theoretically, SAR signals can be characterized as chaotic phenomena since the scattering of signals within a resolution cell can be summed coherently. Accordingly, an SAR signal can be represented by a spatial chaotic model (SCM) and characterized by its fractal dimension. In this study, two approaches for estimating fractal dimensions are conducted, which are estimated by the differential box-counting (DBC) and improved fractal dimension methods in the z-direction. Based on the spatial chaotic model, a simplified SAR image change detection procedure is proposed. This method first calculates the differences in fractal dimensions among multitemporal SAR images to detect the changes in building and grass-recovery areas. Both the constant false alarm rate (CFAR) and support vector machine (SVM) are applied to classify the changed and unchanged areas, respectively. The experimental results reveal that both the DBC and improved fractal dimension methods are similar for detecting changes in building areas. However, regarding the changes in grass recovery areas, the improved fractal dimension method outperforms the DBC method. The results also show that the SVM performs better than the CFAR for both building and grass areas.
\end{abstract}

\section{INTRODUCTION}

Remote sensing is the most feasible approach for largescale land-surface change detection. Change detection techniques have been used successfully in many applications, such as environmental monitoring, land-use/land-cover studies, damage assessments, agricultural surveys, surface deformation and urban change analyses (Chavez and Mackinnon 1994; Bruzzone and Serpico 1997; Hame et al. 1998; Ridd and Liu 1998; Grover et al. 1999; Quegan et al. 2000; Chang et al. 2010). Change detection is a process that analyzes a pair of remote sensing images acquired over the same geographical area at different times in order to identify changes that may have occurred between the considered acquisition dates (Bovolo and Bruzzone 2005). Two main approaches to the change detection problem have been proposed: the supervised approach and unsupervised approach.

\footnotetext{
* Corresponding author

E-mail: sshhnn.heroe@gmail.com
}

The former is based on supervised classification methods, which require the availability of multitemporal ground-truth values to derive a suitable training set. The latter performs change detection by directly comparing multitemporal images. Because the generation of an appropriate multitemporal ground-truth value is usually a difficult task, the use of effective unsupervised change detection methods is fundamental in many applications (Bruzzone and Prieto 2000). $\mathrm{Lu}$ has also provided a comprehensive exploration of all major change detection approaches found in the literature (Lu et al. 2004).

In this paper, we focus our attention on building and grass areas from synthetic aperture radar (SAR) images. It is quite difficult to detect changes directly from SAR images due to the speckle noise associated with SAR sensors. Speckle may be modeled as a correlated signal-dependent random phenomenon that often causes a low signal-to-noise ratio (SNR) in acquired images. Consequently, in the past, a 
speckle reduction algorithm had to be used before a change detection technique could be applied. Due to the intrinsic complexity of the SAR data, both intensive preprocessing and effective data analysis techniques capable of dealing with multiplied speckle noise are required. Many adaptive filters for speckle reduction have been intensively investigated (Lee 1980; Frost et al. 1982; Lopes et al. 1990; Solbø and Eltoft 2004). Singh has also listed several major despeckling methods for SAR images (Singh and Shree 2017).

Change detection in SAR images is usually a three-step procedure: (1) image despeckling, (2) pixelwise comparison of two images, and (3) image thresholding (Bazi et al. 2005; Bovolo and Bruzzone 2005; Tzeng and Chen 2007). Images applying the speckle reduction algorithm result in alterations of the SAR signal to some extent. In spite of its noisy appearance, speckling is indeed an SAR signal naturally. Hence, it can be represented by a spatial chaotic model (SCM). Tzeng and Chen (2007) adopted the differential box-counting (DBC) technique (Sarkar and Chaudhuri 1994) to estimate the fractal dimension of an SAR image because it has been proven to be the least complex computationally, and it is easy to implement. When speckle has been modeled properly, the image despeckling process is no longer required. Therefore, the image despeckling process is substituted with the speckle model estimation in this paper.

Support vector machines are one type of supervised classification for the change detection problem. They can be used in many fields to help solve problems in various applications, especially environmental disasters. The detection of earthquakes and tsunami-induced changes can also utilize this method to detect disasters in the natural environment (Wieland et al. 2016). They can also combined signals from different sources, including microwave and optical images (Zeng et al. 2008). Since the SVM is a supervised classification method, it selects several labeled samples for training to find the hyperplane for a decision boundary, and the remaining labeled samples are used for testing to evaluate the performance. If there are no labeled data available, supervised learning is not possible; therefore, some information about the ground truth data is needed to label samples for the SVM in this study.

In the following section, the spatial chaotic model, DBC, improved fractal dimension method in the z-direction and support vector machine are introduced. Based on the spatial chaotic model, a simplified SAR image change detection procedure is presented in section 3 . Then, this approach is applied to detect changes in building and grass areas in SAR images. Finally, some conclusive remarks are drawn from this study.

\section{METHODOLOGY}

Theoretically, SAR signals can be characterized as chaotic phenomena because the scattered signals within a resolution cell are summed coherently, which has been shown in many studies in the past (Goodman 1976; Leung and Haykin 1990; McDonald et al. 2002; Solbø and Eltoft 2004). This phenomenon in SAR signals can be described by a nonlinear dynamical system. As a result, the modeling of SAR signals becomes a problem for chaotic system reconstructions from time series measurements (Leung et al. 2002). Accordingly, an SAR signal can be represented by a SCM which is briefly introduced in the following section (Tzeng and Chen 2007).

\subsection{Spatial Chaotic Model (SCM) and Differential Box-Counting (DBC)}

A state-space model can describe the dynamics of a nonlinear system (Abarbanel 1996). Let $x_{1}(t), x_{2}(t), \ldots, x_{N}(t)$ denote the state variables of a nonlinear dynamic system, where continuous time $(t)$ is the independent variable, and $N$ represents the order of the system. The form of a system of first-order differential equations is written as follows:

$\frac{d}{d t} x_{i}(t)=F_{i}\left[x_{i}(t)\right]$ for $i=1,2, \ldots, N$

where $F_{i}(\cdot)$ is the nonlinear function of its argument. Fractal dimensions characterize the geometric structure of a strange attractor and define the natural distribution of points for the attractor compared to that of the probability density function for a random variable. According to the details in a reference paper (Tzeng and Chen 2007), the equation can be written as follows:

$C(q, r)=\frac{1}{K} \sum_{n=1}^{K}\left\{\frac{1}{K-1} \sum_{j=1, j \neq n}^{K} H[r-\|x(n)-x(j)\|\}^{q-1}\right.$

where $q$ is an integer and $H$ represents the Heaviside function. The function $C(q, r)$ is the correlation function, which measures the probability of two points $[x(n)$ and $x(j)]$ on the attractor that are separated by a distance of $r$. This correlation function $C(q, r)$ can be rewritten when the number of data points $(K)$ is large and the distance $(r)$ is small.

$C(q, r)=r^{(q-1) D}$

where $D$ represents the fractal dimension of the attractor. As a result, the geometric structure of a chaotic system can be characterized by its fractal dimension for SAR signals.

The DBC technique (Sarkar and Chaudhuri 1994) has proven to be the least computationally complex, and it is easily implemented; therefore, it is adopted in this study to estimate the fractal dimension of SAR images. The basic equation for the fractal dimension $D$ is given by 
$D=\frac{\log \left(N_{r}\right)}{\log (1 / r)}$

In the DBC technique, an image of size $M \times M$ is partitioned into grids of size $s \times s$, where $M / 2 \geq s>1$, and $s$ is an integer. Then, we have an estimation of $r=s / M$. One pixel in the image in 3-D space represents its position on the $x-y$ plane, and the third coordinate $(z)$ denotes its gray level. The $x-y$ plane is partitioned into grids of size $s \times s$. On each grid, there is a column of boxes of size $s \times s \times s^{\prime}$. If the total number of gray levels is equal to $G$, then $s^{\prime}=[\mathrm{G} / \mathrm{M}] \times s$. In grid $(i, j)$, let the minimum and maximum gray levels of the image in the grid be $g_{l}$ and $g_{u}$, respectively. The number of boxes between the minimum and maximum gray levels at grid $(i, j)$ is counted by

$n_{r}(i, j)=g_{u}-g_{l}+1$

Due to the differential nature of computing $n_{r}$, this method is called the differential box-counting approach. Therefore, the total number of boxes in the whole region of interest is simply the summation of the number of boxes in all grids

$N_{r}=\sum_{i, j} n_{r}(i, j)$

$N_{r}$ is calculated for different values of $r$ (i.e., different values of grid size $s$ ). The reason for counting $N_{r}$ is to provide a better coverage of boxes for the intensity range of the image. In a sense, the DBC approach makes an approximation for the difference in box height.

\subsection{Improved Fractal Dimension}

Sarkar and Chaudhuri (1994) showed that the DBC is among the best in terms of efficiency and dynamic range for fractal dimensions (FDs), but it has a lower accuracy. Some disadvantages of the DBC have also been discussed in other studies (Chen et al. 2003). They indicated some problems, including that the DBC may overcount the number of boxes covering the image intensity surface. Box counting does not precisely capture the estimation of the fractal dimension because it does not cover the image intensity surface sufficiently enough, especially for high-changing surface images. If we only need three boxes to represent a high variation range, we do not need more boxes. As shown in the example in Fig. 1, if the boxes are appropriately shifted along the zdirection, only three boxes rather than four boxes are sufficient to demonstrate the gray-level variation in the intensity surface for the column of boxes. When the boxes are shifted along the z-direction, the improved number of boxes covering the image intensity surface should be no greater than the original number, which can be easily shown below.

ceil $\left(\frac{g_{u}-g_{l}+1}{s}\right) \leq \operatorname{ceil}\left(\frac{g_{u}}{s}\right)-$ floor $\left(\frac{g_{l}}{s}\right)$

$n_{r}(i, j)=\operatorname{ceil}\left(\frac{g_{u}-g_{l}+1}{s}\right)$

The function ceil rounds a number to the next largest integer. Therefore, in this paper, we calculate the improved fractal dimension for comparison with the original DBC and intensity image.

\subsection{Support Vector Machine (SVM)}

Support vector machines (SVMs) are a kind of machine learning technique (Burges 1998). They can be applied to analyze data via regression and classification methods. In the case of classification, a hyperplane is generated to separate different class groups for multidimensional features in

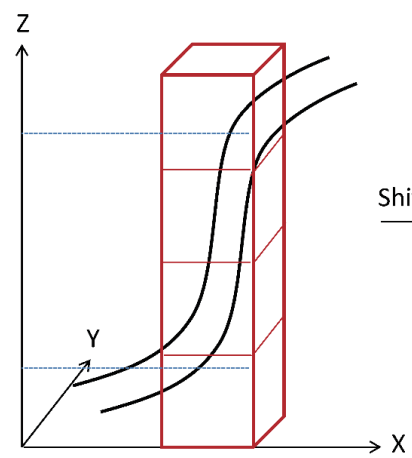

original fractal dimension

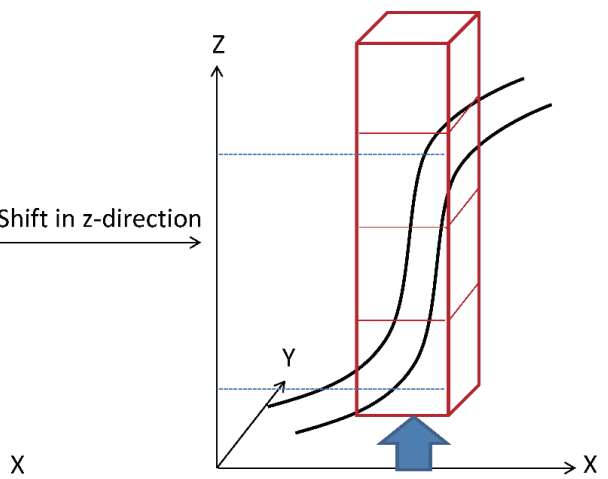

improved fractal dimension

Fig. 1. Estimation approach via the original fractal dimension and improved fractal dimension methods. 
high-dimensional space. To obtain the best decision boundary, it is important to find the optimal hyperplane to separate labeled samples from different classes with the maximum margins. Given a particular hyperplane, we compute the distance from the hyperplane to the closest data point. The value that is twice this value is called the margin. In this study, we consider a nonlinear case.

Given a training set of $N s$ data points, the sign of $f(x)$ gives the label of the sample.

$$
f(x)=\operatorname{Sign}\left(\sum_{i=1}^{N s} y_{i} \cdot \alpha_{i}\left\langle x, x_{i}\right\rangle+b\right)
$$

where $\alpha_{i}$ is the Lagrangian multiplier (i.e., support value), $b$ represents the bias, Ns represents the set of support vectors, and $y_{i}$ represents the class of data point $i(+1$ or -1$)$. The value of $f(x)$ denotes the distance in the tested instance from the separated hyperplane, and the sign indicates the class label.

The kernel is a function that simulates the projection of initial data onto a feature space with a higher dimension. There are many kernel functions available; however, a nonlinear SVM with a Gaussian radial basis function (RBF) is adopted in this study since it is the most popular kernel method among other studies on SAR data (Akbarizadeh 2012; Yekkehkhany et al. 2014) due to the relevance of the RBF kernel to SAR data, which has a Gaussian contribution.

$\mathrm{k}\left(\bar{x}_{i}, \bar{x}_{j}\right)=\exp \left(-\gamma \cdot\left\|\bar{x}_{i}-\bar{x}_{j}\right\|^{2}\right)$

\section{RESULTS}

The improved fractal estimation in the z-direction compared with the intensity and DBC methods via the CFAR and SVM for SAR image change detection without a speckle reduction process is displayed in Fig. 2. The algorithm is summarized below:

(1) Coregister the multitemporal image sets.

(2) Estimate the fractal dimension of each data set with a moving window $(M=9)$ and grid size $(s=3)$ for both the DBC and improved fractal dimension methods.

(3) Take the difference in the two fractal dimension images.

(4) Perform the CFAR and SVM detections.

(5) Evaluate the detection performance with Eq. (11).

$$
\begin{aligned}
& \mathrm{DR}=\frac{A_{c}}{A_{s}} \\
& \mathrm{FDR}=\frac{A_{d}-A_{c}}{A_{u n}} \\
& \mathrm{LDR}=\frac{A_{s}-A_{c}}{A_{s}}
\end{aligned}
$$

The experimental results of the proposed approach were compared to those via the difference image (DI) method as reference. The detection performance was assessed by three detection evaluation measures: the detection rate (DR), which denotes the accuracy rate of a detected changed target; the false detection rate (FDR), which denotes the false alarm rate of a target that is actually unchanged but is identified as changed; and the loss detection rate (LDR), which denotes the missing rate of a target that has actually changed but is identified as unchanged. Let Standard Area $\left(A_{s}\right)$ denote the target change area on the ground truth map, Unchanged Area $\left(A_{u n}\right)$ denote the area without change on the ground truth map, Detected Area $\left(A_{d}\right)$ denote the areas classified as changed by the detection algorithm, and Correct Area $\left(A_{c}\right)$ denote the intersection region of the Standard Area and Detected Area. The detection rate (DR), false detection rate (FDR), and loss detection rate (LDR) detection measures are adopted from another paper (Qu et al. 2003), with a modification to the FDR that is more intuitive. The CFAR method is carried out by select a threshold so that the false alarm rate is less than a pre-determined constant while maximize the detection rate. In this paper, the definition of FDR is the same as false alarm rate, and LDR $=1$ - DR.

Before the receiver operating characteristic (ROC) curve was employed in the signal detection theory, it was first used during World War II for the analysis of radar signals (Green and Swets 1966). An ROC curve is the most commonly used approach to diagnose the evaluation of a binary classifier system, as its threshold value varies. The perfect detection occurs when the ROC curve reaches the top left corner (i.e., where DR $=1$ and FDR $=0$ ). The advantage of the ROC curve is that it can also determine optimal threshold values.

\subsection{Study Area}

The SAR images for the experiments are collected by Terra SAR-X, an Earth radar observation satellite at the Xband (wavelength $31 \mathrm{~mm}$, frequency $9.6 \mathrm{GHz}$ ). Because of their long wavelengths compared to the visible spectrum, microwaves can penetrate cloud cover, and they are less susceptible to the heaviest rainfall. Therefore, microwave properties allow for detection under almost all-weather conditions. Staelin measured atmospheric absorption spectra and showed the relationship between frequency and zenith attenuation (Staelin 1966). The polarization mode is the strip map for dual polarization. This satellite is a high-resolution radar satellite that has a spatial resolution up to $1 \mathrm{~m}$. At a 514-km altitude, it passes nearly above both poles on Earth. This image scene is taken with an ascending orbit and looking to the right.

Both horizontal-horizontal $(\mathrm{HH})$ and horizontal-vertical (HV) polarization of the Terra SAR-X on 14 August 2009 and 21 June 2010, are used for the experiments. The image 
scene is located in the Jiaxian township (Kaohsiung County) in southern Taiwan. The ground truth samples for the building and grass areas from the two test sites are selected for the performance evaluation. The buildings are prefabricated houses built on a flat area near the river. Figure $3 \mathrm{a}$ depicts an SAR image for intensity via HH polarization acquired on 14 August 2009. Figure 3b shows an SAR image for intensity via $\mathrm{HH}$ polarization acquired on 21 June 2010 , after rebuilding and vegetation recovery. In this study, an accuracy assessment is conducted for change detection in two test areas via the CFAR and SVM methods for the DI for intensity, the fractal dimension via the $\mathrm{DBC}$ and the improved fractal dimension methods. A landslide caused by Typhoon Morakot on 8 August 2009, is observed in the study area. Although

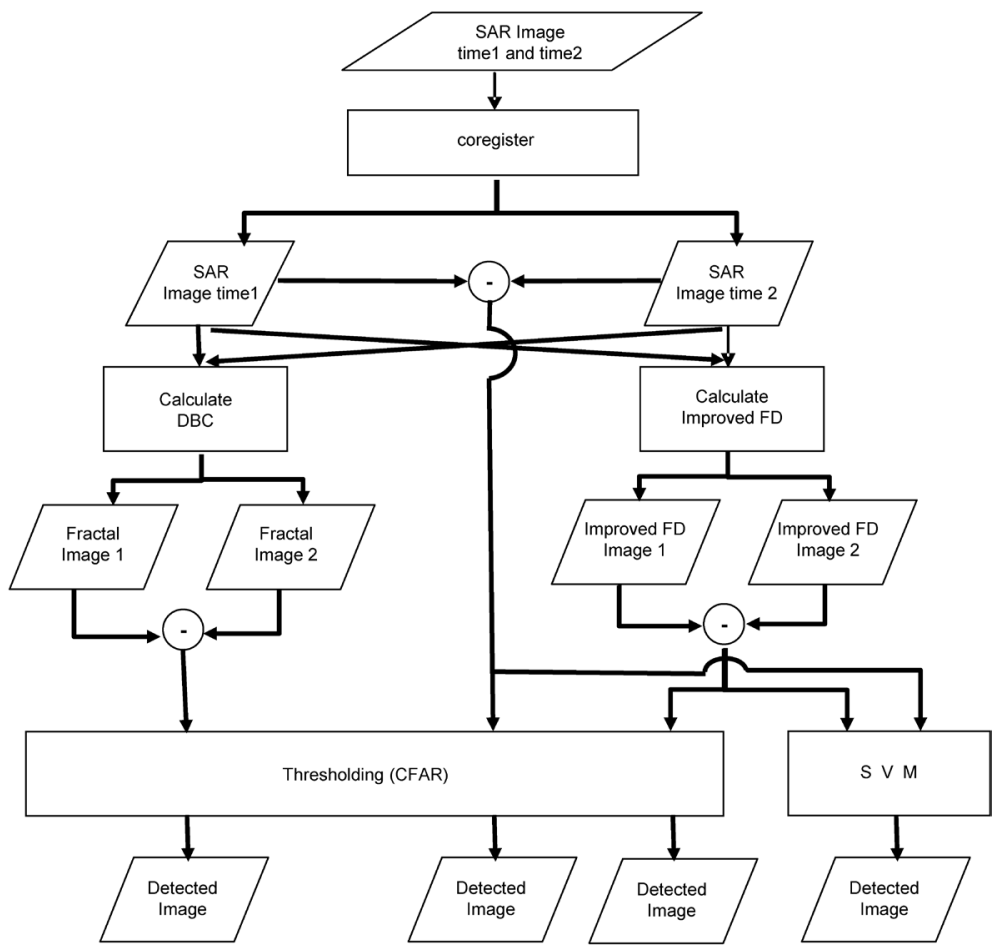

Fig. 2. Flow chart of the proposed SAR image change detection procedure.

(a)

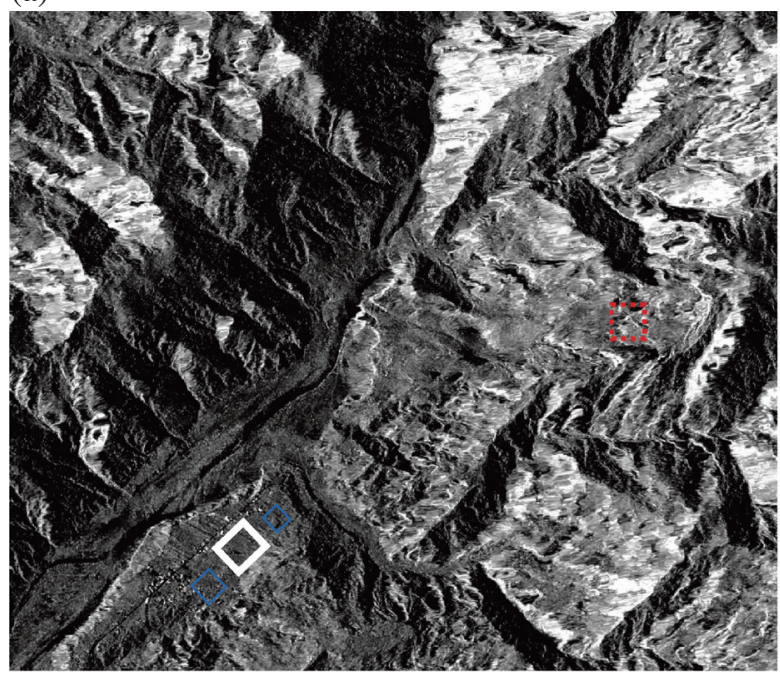

(b)

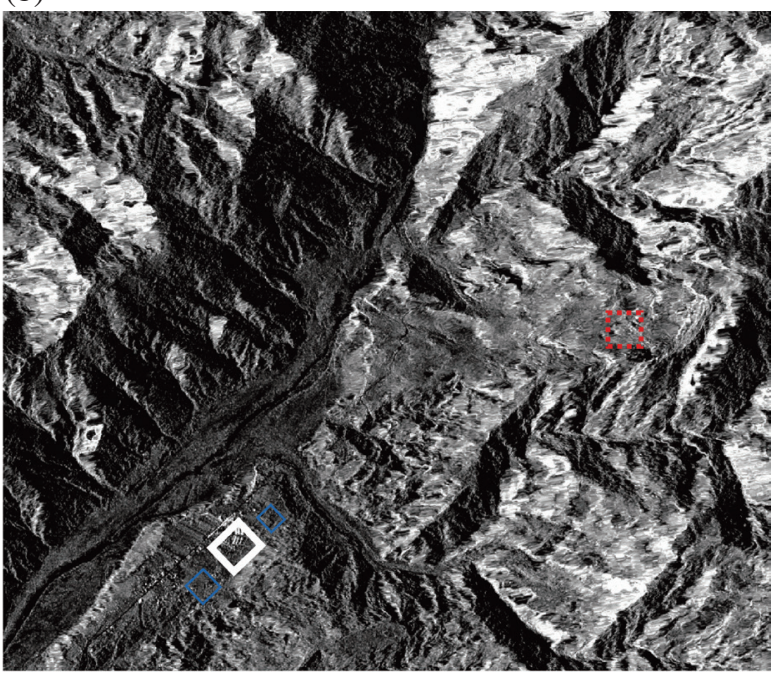

Fig. 3. (a) The HH intensity SAR images show us a topographic map on 14 October 2009 that points out the changed area for buildings, changed area for grass and unchanged areas, which are indicated by white (thick lines), red (dashed lines), and blue (fine lines) boxes. (b) The HH intensity SAR images show us a topographic map on 21 June 2010 that points out the changed area for buildings, changed area for grass and unchanged areas, which are indicated by white (thick lines), red (dashed lines), and blue (fine lines) boxes. 
this area contains buildings, trees, rivers, and grass, the changes in buildings and the grass recovery were observed in the field survey on 12 November 2010. Therefore, building and grass areas are selected as the main focus in our experiments. Figure 4 is an optical satellite image of test site on 29 January 2010 from Google Maps as a reference image.

In addition to the CFAR, both the HH and HV bands are considered to detect changes in intensity and the fractal and improved fractal dimensions, respectively. A pixel is classified as a changed pixel if and only if that pixel has been classified changed in all bands of the image via the CFAR. The intensity bands are calculated from the real and imaginary parts of the raw data in the SAR image. Based on the intensity, we estimate the fractal and improved fractal dimensions for change detection in this study. The SVM considers four bands: HH intensity, HV intensity, HH improved fractal dimension, and HV improved fractal dimension. We randomly select $50 \%$ samples for training, and the rest are used for testing for the test area in this experiment.

The provided SAR image for intensity via $\mathrm{HH}$ polarization is illustrated in Fig. 3; the ground truth data for the changed areas of buildings and grass and the unchanged areas are indicated by white (thick lines), red (dashed lines), and blue (fine lines) boxes, respectively.

\subsubsection{Test Site 1}

In Fig. 3a, the village was damaged by a landslide after the disaster. Figure $3 \mathrm{~b}$ shows that the reconstruction of the prefabricated house was built on a flat area after one year and appeared as a bright area. A comparison of the detection performances was conducted for the DI for intensity, fractal dimension $[9,3]$ and improved fractal dimension via the CFAR and other methods [i.e., SVM, including 4 bands with the receiver operating characteristic (ROC) curve] and are shown in Fig. 5. The fractal dimension $[9,3]$ indicates a moving window of $M=9$ and a grid size of $s=3$, which perform well in our experiments. The fractal dimension of the DBC, the improved fractal dimension and the SVM all show good performances, with a very high detection rate and very low false detection rate. The DI for intensity is the worst among them. As shown in Fig. 5, the DR and FDR for the improved fractal dimension are similar to the original fractal dimensions. It does not show much improvement compared to the original fractal dimensions for detecting buildings. This is the main reason that the DI cannot distinguish the ROC curve for the original fractal dimensions, improved fractal dimensions and SVM when detecting buildings, but they are all much better than the DI for intensity.

\subsubsection{Test Site 2}

In the second test area, part of the grass in the SAR image in Fig. 3a was removed after the landslide, and the grass grew back after a few months. The grass area is marked with a red box in Fig. 3b. The ROC curves for the grass area by using these approaches are drawn for an accuracy comparison. As in Fig. 6, the improved fractal dimensions outperformed the original fractal method. However, the intensity method results are better than the fractal results $[9,3]$, which can be observed in the histogram for fractal dimension formed from ground truths in Fig. 7. The samples from the unchanged area are highly overlapped with the changed area. However, the histogram for intensity in Fig. 8 shows that some differences in the histogram can be observed between changed and unchanged areas in band 1,

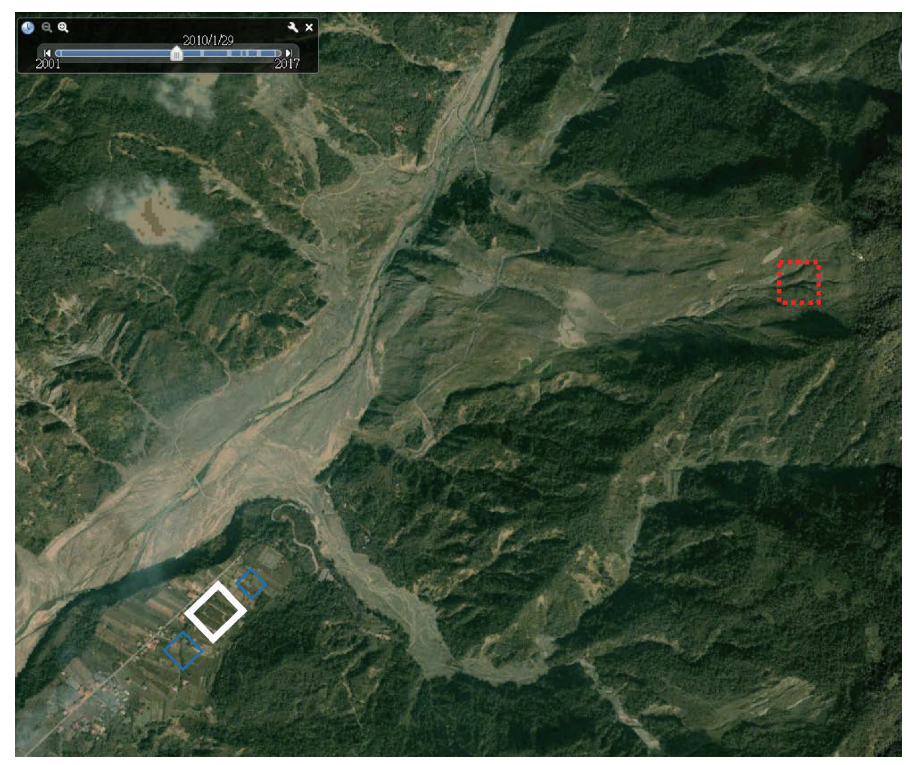

Fig. 4. Ground truths via Google Maps on 29 January 2010. 


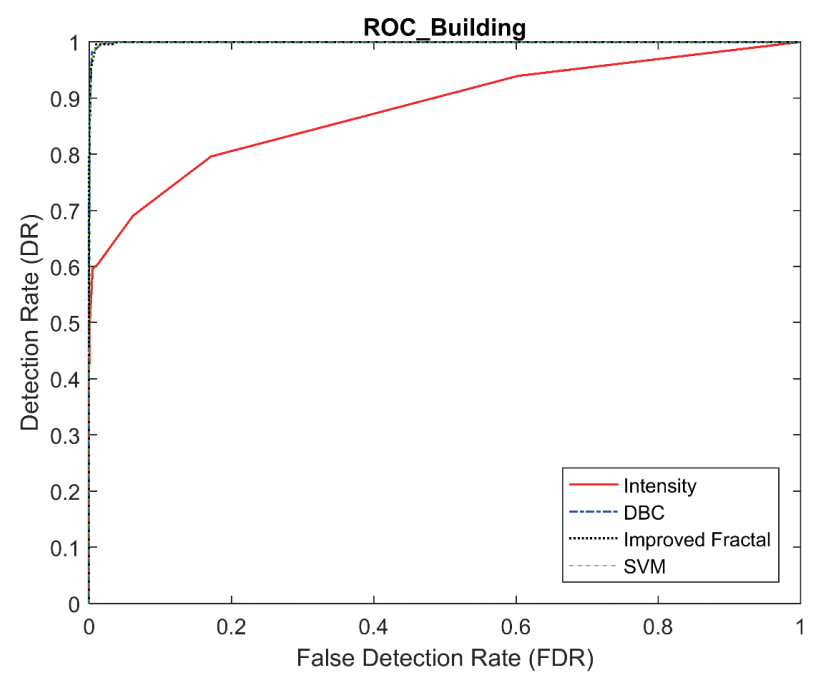

Fig. 5. ROC curve via the intensity, DBC, improved fractal and SVM methods for building areas.

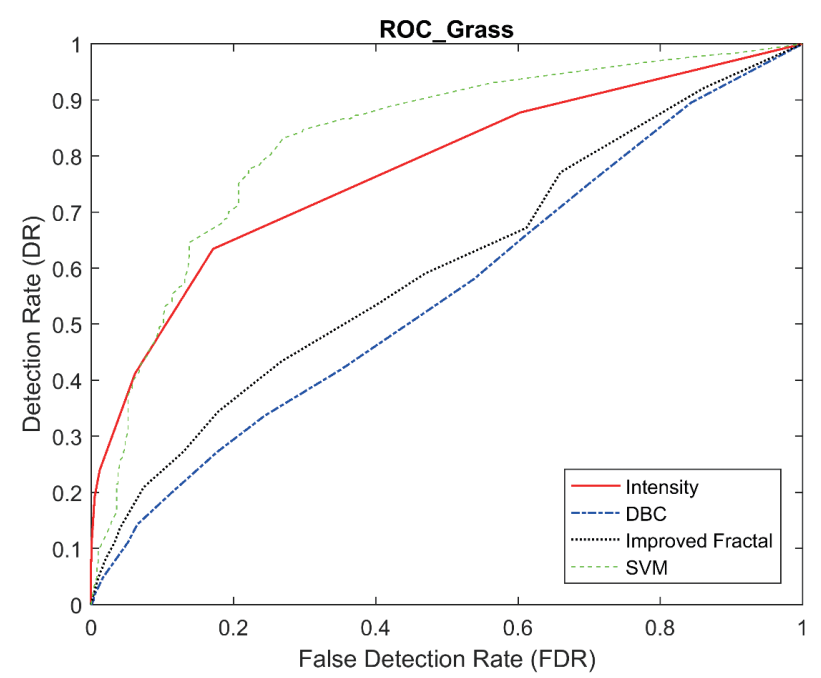

Fig. 6. ROC curve via the intensity, DBC, improved fractal and SVM methods for grass area.
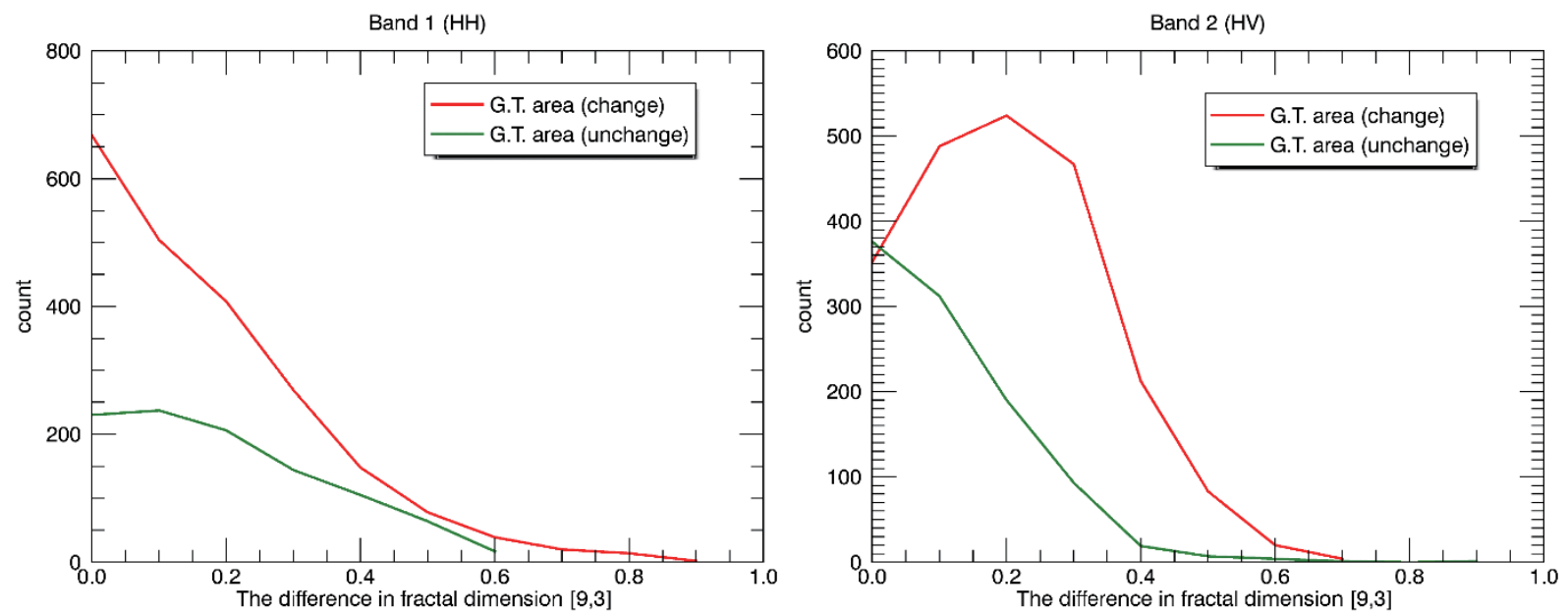

Fig. 7. A histogram of different fractal dimensions for grass in changed and unchanged areas via the ground truth values using different polarizations (HH and $\mathrm{HV})$. 

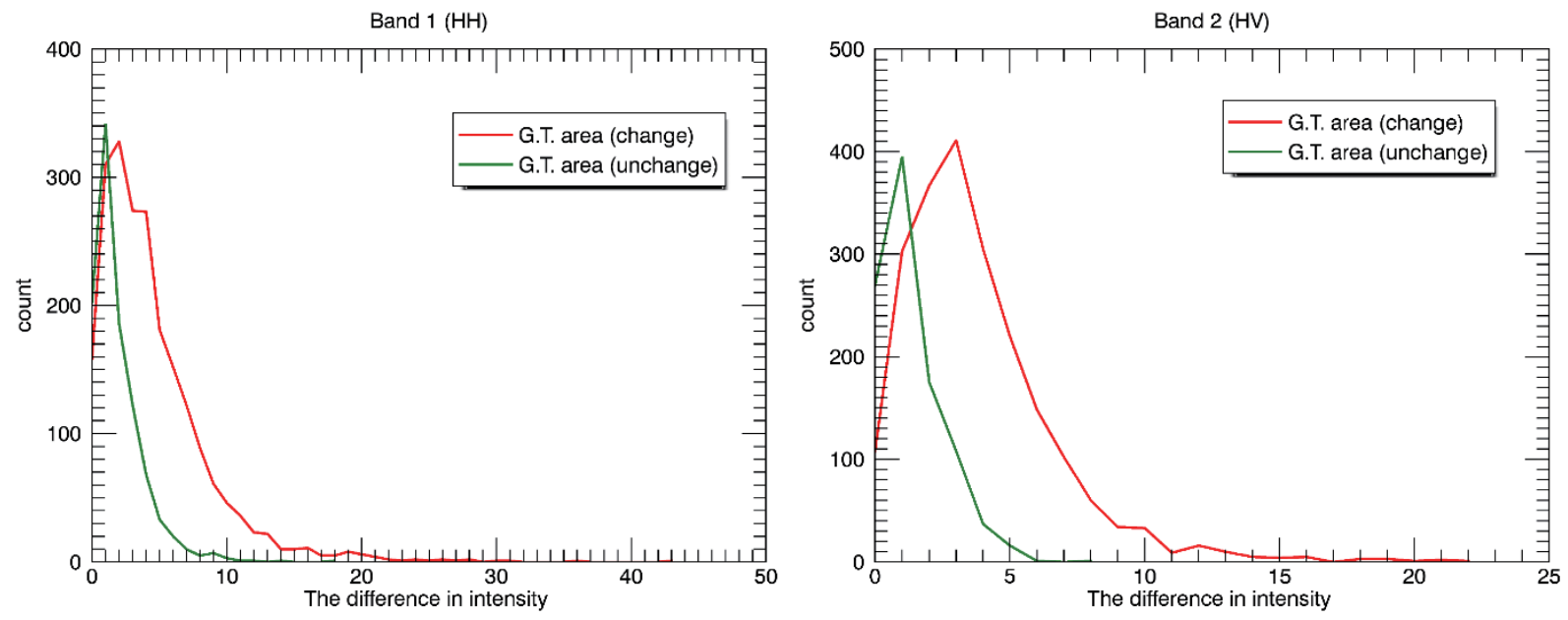

Fig. 8. A histogram of different intensities for grass in changed and unchanged areas via the ground truth values using different polarizations (HH and $\mathrm{HV}$ ).

and the peaks in band 2 have better separation than those in band 1. Thus, change detection for the grass area cannot be easily recognized by the fractal dimension approach only. To improve this performance, the SVM is conducted to separate the two areas with a hyperplane. With the four bands of $\mathrm{HH}$ intensity, $\mathrm{HV}$ intensity, $\mathrm{HH}$ improved fractal dimension, and HV improved fractal dimension, the ROC curve of the SVM shows the best performance among these four approaches. The ground truth values for the unchanged and changed areas are shown in Fig. 9, and Fig. 10 shows the change detection results of the test areas. The probability of the FDR is chosen to be $5 \%$ based on the unchanged samples in the training set for intensity and fractal and improved fractal dimension cases. The SVM method is conducted with the libSVM software. The white pixels indicate the changes between the SAR images from different dates, and the black pixels indicate those that occurred in the unchanged areas. Table 1 shows the results when the threshold is set with a fixed FDR of 5\% for the unchanged area from the training set. This threshold is then applied to the testing area to calculate the DR and FDR; therefore, the FDR in Table 1 varies from $0.1 \%$ (intensity difference) to $37 \%$ (SVM). In Table 2, the same procedure is conducted, but the threshold is set with a fixed FDR of 5\% from the unchanged samples in the testing set, which are used directly as baselines for comparison. Both tables indicate that the improved fractal dimension outperforms the original fractal dimension for both building and grass sites. However, compared to the intensity feature, the DRs for both fractal dimension features are significantly better than those for intensity in the building sites but opposite those in the grass sites. By combining intensity and the improved fractal dimension features via the SVM, the accuracy is comparable to that of the fractal dimension for building sites but significantly improved for the grass sites.

\section{DISCUSSION}

There are several studies that have performed target detection based on fractal dimension. Our experimental results show that this feature is excellent for detecting buildings to eliminate noise or speckle without applying filters or other enhancement techniques. In addition, the ROC curve is very close to the upper-left corner, which indicates that the detection result is better than the others. The proposed improved fractal method performs similarly to the original fractal dimension when detecting building areas, but it has a significant improvement in grass areas. The main reason that the DR for the fractal dimension is worse than that for the intensity feature in grass sites is discussed. It can be easily observed that the histogram for the intensity feature can be obviously distinguished in changed and unchanged grass areas, especially in the HV polarization band. However, in the histogram for fractal dimensions, both changed and unchanged areas are similar for both grass and building areas, especially for noise and speckle. The ROC curves show that the improved fractal dimension is better than the original fractal feature, and an SVM with intensity and improved fractal dimensions has the best accuracy for most of the FDRs. Only when the FDR is less than $4 \%$ is the SVM slightly worse than the intensity feature in the grass area. The changed building area does not show a significant difference between the improved and original fractal dimensions in the ROC curve. In the detected results, the detected area is also similar. This is because the difference in estimated value between the two fractal dimensional approaches is small compared to the intensity difference for strong scatterers (i.e., buildings). A threshold value of $5 \%$ for the FDR is set for the training and testing areas, and the DR and FDR of the testing area are calculated in Tables 1 and 2, respectively. Therefore, the detection rates 

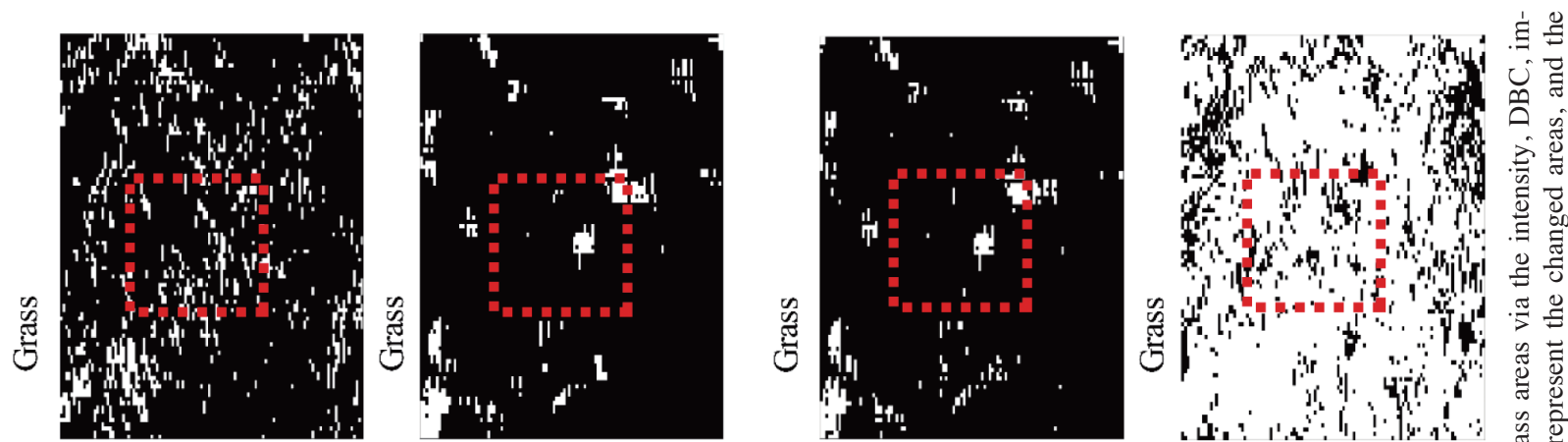

की

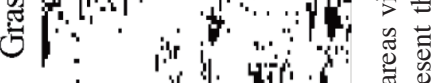

1.
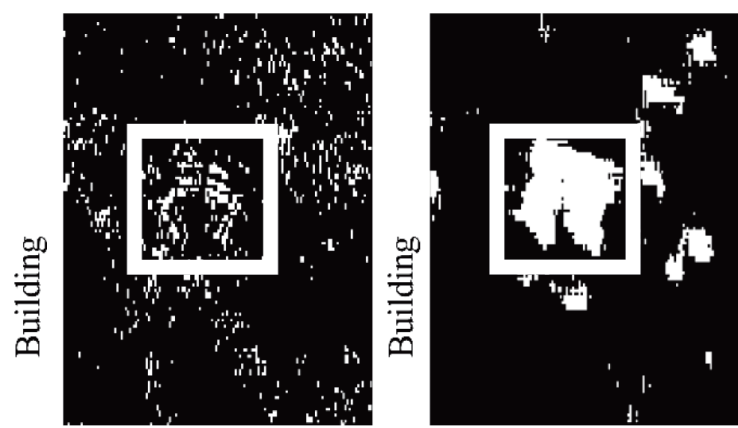

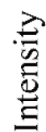

宽

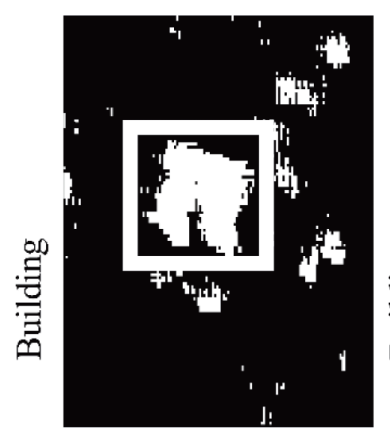

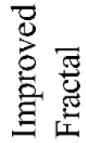

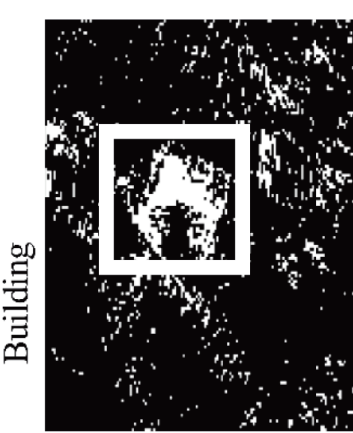

就

承 $\stackrel{\text { OD }}{\Xi}$ 를

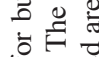
$\triangleq \dot{0}$ के उ $\frac{\mathscr{\sigma}}{\sum} \sum$ 矛的苍 . 픈 E 으믈 .
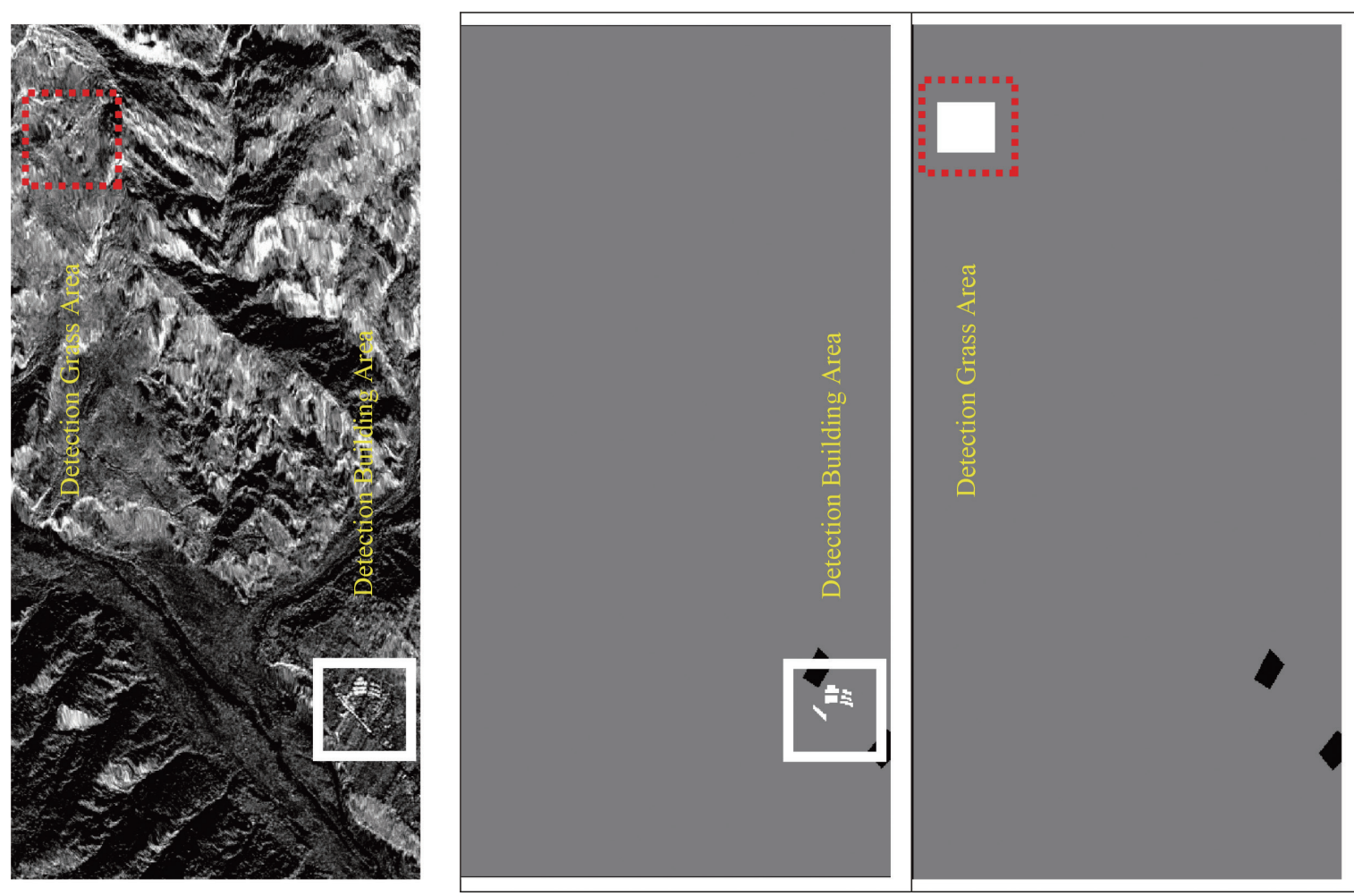

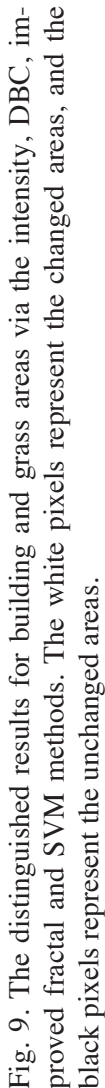


Table 1. The results when the threshold is set with a fixed FDR of 5\% for the unchanged area from the training set.

\begin{tabular}{ccccc}
\hline Building & Intensity & Fractal & Improved Fractal & SVM \\
\hline Detection Rate & $50.2 \%$ & $99.6 \%$ & $99.6 \%$ & $97.0 \%$ \\
False Detection Rate & $0.1 \%$ & $1.7 \%$ & $1 \%$ & $0.2 \%$ \\
Loss Detection Rate & $49.8 \%$ & $0.4 \%$ & $0.4 \%$ & $3.0 \%$ \\
\hline Grass & Intensity & Fractal & Improved Fractal & SVM \\
\hline Detection Rate & $11.5 \%$ & $4.9 \%$ & $4.7 \%$ & $86.5 \%$ \\
False Detection Rate & $0.1 \%$ & $1.7 \%$ & $1 \%$ & $37.4 \%$ \\
Loss Detection Rate & $88.5 \%$ & $95.1 \%$ & $95.3 \%$ & $13.5 \%$ \\
\hline
\end{tabular}

Table 2. The results when the threshold is set with a fixed FDR of 5\% for the unchanged area from the testing set.

\begin{tabular}{ccccc}
\hline Building & Intensity & Fractal & Improved Fractal & SVM \\
\hline Detection Rate & $50.2 \%$ & $98.5 \%$ & $99.0 \%$ & $97.6 \%$ \\
False Detection Rate & $0.5 \%$ & $0.5 \%$ & $0.5 \%$ & $0.5 \%$ \\
Loss Detection Rate & $49.8 \%$ & $1.5 \%$ & $1.0 \%$ & $2.4 \%$ \\
\hline Grass & Intensity & Fractal & Improved Fractal & SVM \\
\hline Detection Rate & $37.7 \%$ & $11.1 \%$ & $16.3 \%$ & $31.2 \%$ \\
False Detection Rate & $0.5 \%$ & $0.5 \%$ & $0.5 \%$ & $0.5 \%$ \\
Loss Detection Rate & $62.3 \%$ & $88.9 \%$ & $83.7 \%$ & $68.8 \%$ \\
\hline
\end{tabular}

are quite different for grass, but both tables indicate the improved performance of the improved fractal dimension and SVM methods.

\section{CONCLUSION}

A simple SAR image change detection procedure is presented based on the spatial chaotic model and improved fractal dimension method via the CFAR and SVM. Different methods are applied for a performance comparison between building and grass areas. The experimental results reveal that both fractal dimension methods are effective and more efficient than intensity for estimating building areas. The improved fractal dimension method is better than the original fractal dimension method for estimating grass areas from SAR images. For both building and grass areas, the experiment shows that the SVM with both HH and HV polarization for intensity and improved fractal dimensions has the best classification result. Therefore, both intensity and improved fractal dimension data should be considered for change detection in SAR images. The proposed method has demonstrated its capability for detecting change by combining intensity and improved fractal dimensions via the SVM with a dual-polarized SAR image. This method can be further extended for full polarization if $\mathrm{HH}, \mathrm{HV}, \mathrm{VH}$, and $\mathrm{VV}$ are available and more features can be included based on the target properties.

\section{REFERENCES}

Abarbanel, H. D. I., 1996: Analysis of Observed Chaotic Data, Springer-Verlag New York, 272 pp, doi: 10.1007/978-1-4612-0763-4. [Link]

Akbarizadeh, G., 2012: A New Statistical-Based Kurtosis Wavelet Energy Feature for Texture Recognition of SAR Images. IEEE Trans. Geosci. Remote Sensing, 50, 4358-4368, doi: 10.1109/tgrs.2012.2194787. [Link]

Bazi, Y., L. Bruzzone, and F. Melgani, 2005: An unsupervised approach based on the generalized Gaussian model to automatic change detection in multitemporal SAR images. IEEE Trans. Geosci. Remote Sensing, 43, 874-887, doi: 10.1109/tgrs.2004.842441. [Link]

Bovolo, F. and L. Bruzzone, 2005: A detail-preserving scaledriven approach to change detection in multitemporal SAR images. IEEE Trans. Geosci. Remote Sensing, 43, 2963-2972, doi: 10.1109/tgrs.2005.857987. [Link]

Bruzzone, L. and D. F. Prieto, 2000: Automatic analysis of the difference image for unsupervised change detection. IEEE Trans. Geosci. Remote Sensing, 38, 11711182, doi: 10.1109/36.843009. [Link]

Bruzzone, L. and S. B. Serpico, 1997: An iterative technique for the detection of land-cover transitions in multitemporal remote-sensing images. IEEE Trans. Geosci. Remote Sensing, 35, 858-867, doi: 10.1109/36.602528. [Link] 
Burges, C. J. C., 1998: A Tutorial on Support Vector Machines for Pattern Recognition. Data Min. Knowl. Discov., 2, 121-167, doi: 10.1023/A:1009715923555. [Link]

Chang, C.-P., J.-Y. Yen, A. Hooper, F.-M. Chou, Y.-A. Chen, C.-S. Hou, W.-C. Hung, and M.-S. Lin, 2010: Monitoring of Surface Deformation in Northern Taiwan Using DInSAR and PSInSAR Techniques. Terr. Atoms. Ocean. Sci., 21, 447-461, doi: 10.3319/ TAO.2009.11.20.01(TH). [Link]

Chavez, P. S. and D. J. Mackinnon, 1994: Automatic Detection of Vegetation Changes in the Southwestern United States Using Remotely Sensed Images. Photogrammetric Engineering and Remote Sensing, 60, 571-583.

Chen, W. S., S. Y. Yuan, and C. M. Hsieh, 2003: Two algorithms to estimate fractal dimension of gray-level images. Opt. Eng., 42, 2452-2464, doi: 10.1117/1.1585061. [Link]

Frost, V.S.,J.A. Stiles, K. S.Shanmugan, and J.C.Holtzman, 1982: A Model for Radar Images and Its Application to Adaptive Digital Filtering of Multiplicative Noise. IEEE Trans. Pattern Anal. Mach. Intell., PAMI-4, 157-166, doi: 10.1109/TPAMI.1982.4767223. [Link]

Goodman, J. W., 1976: Some fundamental properties of speckle. J. Opt. Soc. Am., 66, 1145-1150, doi: 10.1364/ josa.66.001145. [Link]

Green, D. M. and J. A. Swets, 1996: Signal Detection Theory and Psychophysics, Wiley, New York, 30-52.

Grover, K., S. Quegan, and C. da Costa Freitas, 1999: Quantitative estimation of tropical forest cover by SAR. IEEE Trans. Geosci. Remote Sensing, 37, 479-490, doi: 10.1109/36.739096. [Link]

Hame, T., I. Heiler, and J. San Miguel-Ayanz, 1998: An unsupervised change detection and recognition system for forestry. Int. J. Remote Sens., 19, 1079-1099, doi: 10.1080/014311698215612. [Link]

Lee, J. S., 1980: Digital Image Enhancement and Noise Filtering by Use of local Statistics. IEEE Trans. Pattern Anal. Mach. Intell., PAMI-2, 165-168, doi: 10.1109/ tpami.1980.4766994. [Link]

Leung, H. and S. Haykin, 1990: Is there a radar clutter attractor? Appl. Phys. Lett., 56, 593-595, doi: 10.1063/1.102750. [Link]

Leung, H., N. Dubash, and N. Xie, 2002: Detection of small objects in clutter using a GA-RBF neural network. IEEE Trans. Aero. Electron. Syst., 38, 98-118, doi: 10.1109/7.993232. [Link]

Lopes, A., E. Nezry, R. Touzi, and H. Laur, 1990: Maximum a Posteriori Speckle Filtering and First Order Texture Models in SAR Images. 10th Annual International Symposium on Geoscience and Remote Sensing, College Park, Maryland, USA, 2409-2412, doi: 10.1109/IGARSS.1990.689026. [Link]

Lu, D., P. Mausel, E. Brondízio, and E. Moran, 2004:
Change Detection Techniques. Int. J. Remote Sens., 25, 2365-2401, doi: 10.1080/0143116031000139863. [Link]

McDonald, M. K., V. Varadan, and H. Leung, 2002: Chaotic behaviour and non-linear prediction of airborne radar sea clutter data. Proceedings of the 2002 IEEE Radar Conference (IEEE Cat. No.02CH37322), IEEE, Long Beach, CA, USA, 331-337, doi: 10.1109/ NRC. 2002.999740. [Link]

Qu, J., C. Wang, and Z. Wang, 2003: Structure-context based fuzzy neural network approach for automatic target detection. Proceedings of the 2003 IEEE International Geoscience and Remote Sensing Symposium (IEEE Cat. No.03CH37477), IEEE, Toulouse, France, 767-769, doi: 10.1109/IGARSS.2003.1293911. [Link]

Quegan, S., T. Le Toan, J. J. Yu, F. Ribbes, and N. Floury, 2000: Multitemporal ERS SAR analysis applied to forest mapping. IEEE Trans. Geosci. Remote Sensing, 38, 741-753, doi: 10.1109/36.842003. [Link]

Ridd, M. K. and J. Liu, 1998: A Comparison of Four Algorithms for Change Detection in an Urban Environment. Remote Sens. Environ., 63, 95-100, doi: 10.1016/ s0034-4257(97)00112-0. [Link]

Sarkar, N. and B. B. Chaudhuri, 1994: An efficient differential box-counting approach to compute fractal dimension of image. IEEE Trans. Syst. Man. Cybern., 24, 115-120, doi: 10.1109/21.259692. [Link]

Singh, P. and R. N. Shree, 2017: Statistical Quality Analysis of Wavelet Based SAR Images in Despeckling Process. Asian Journal of Electrical Sciences, 6, 1-18.

Solbø, S. and T. Eltoft, 2004: Homomorphic wavelet-based statistical despeckling of SAR images. IEEE Trans. Geosci. Remote Sensing, 42, 711-721, doi: 10.1109/ tgrs.2003.821885. [Link]

Staelin, D. H., 1966: Measurements and Interpretation of the microwave spectrum of the terrestrial atmosphere near 1-centimeter wavelength. J. Geophys. Res., 71, 2875-2881, doi: 10.1029/jz071i012p02875. [Link]

Tzeng, Y.-C. and K.-S. Chen, 2007: Change detection in synthetic aperture radar images using a spatially chaotic model. Opt. Eng., 46, doi: 10.1117/1.2771146. [Link]

Wieland, M., W. Liu, and F. Yamazaki, 2016: Learning Change from Synthetic Aperture Radar Images: Performance Evaluation of a Support Vector Machine to Detect Earthquake and Tsunami-Induced Changes. Remote Sens., 8, doi: 10.3390/rs8100792. [Link]

Yekkehkhany, B., A. Safari, S. Homayouni, and M. Hasanlou, 2014: A Comparison Study of Different Kernel Functions for SVM-Based Classification of MultiTemporal Polarimetry SAR Data. Int. Arch. Photogramm. Remote Sens. Spatial Inf. Sci., XL-2/W3, 281285, doi: 10.5194/isprsarchives-XL-2-W3-281-2014. [Link] 
Zeng, Y., J. Zhang, and J. L. Van Genderen, 2008: Change detection approach to SAR and optical image integration. ISPRS 2008: Proceedings of the XXI Congress:
Silk Road for Information from Imagery: The International Society for Photogrammetry and Remote Sensing, 3-11 July, Beijing, China, 1077-1083. 